\title{
CAPACITY BUILDING FOR SUSTAINABLE DEVELOPMENT
}

\author{
WIJAYA JAYATILAKA \\ Director, Sri Lanka Foundation Institute, 100, Independence Square, Colombo 7, \\ Sri Lanka
}

\section{Proceedings of the South and Central Asian MAB Meeting of Experts on Environmental Conservation, Management and Research, Hikkaduwa, Sri Lanka 15-18 October 2002}

Summary: A judicious balance between meeting the needs and expectations of the present generation of people without jeopardizing the quality of the environment, or the ability to meet the needs of future generations, will be the ideal situation that may ensure sustainability. Such a scenario assumes that the society is able to cope with any unforeseen challenges that may place severe constraints on resource requirements. Such challenges may be due to man made or natural disasters and changes in consumption habits. The challenges faced are thus to ensure that there is sustainability, of not only resource use, but also improvements of the resource base so that future challenges are met with ease. The capacity to do so will depend on the ability to monitor and assess resource requirements, and respond effectively without undue social or environmental strain.

Development across the globe has resulted in ever rising expectations and struggles to meet requirements. These efforts are addressed by various social institutions. Primary among these is the government. Other institutions are family, community organizations, law and its enforcement agencies, media, education, etc. These institutions are both local and external and the latter can be within the country as well as international.

Acute poverty and inequalities undermine peace and affects sustainable development. Ensuring human security with adequate supply of food, water and livelihoods to all people are considered essential and is a collective responsibility of all nations. Increasing population and changing consumption patterns which are inequitably distributed adds strain to the international community. Population growth in developing countries may exceed their carrying capacity. The consumption patterns of the developed countries also seem to exceed global capacities. A healthy environment is a pre-requisite to good health and is directly related to sustainability. Judicious management of resources through capable institutions is imperative.

Protecting the environment will entail addressing issues affecting the atmosphere, land use, deforestation, desertification, mountain development, agriculture, biodiversity, biotechnology, ocean and fresh water management as well as safe use of toxic chemicals. All these issues are addressed by different social institutions distributed from the international to local levels. Human interventions are carried out at different levels namely, family, community, regions, national as well as global. These institutions not only act on their own capacity but link up and form networks to carry out their functions. The capacity of the institutions to deal with the challenges depends on the skills, work culture, standards, and resource base. Local and global initiatives help develop institutional capacity. Such interventions need to be done at the individual level which will entail skill development 
and at the organizational level, developing and institutionalizing work norms and standards. Developing the external institutional environment which includes the policy framework, legal systems, communication facilities, training and education systems, and inter organizational working relationships are also required.

At an organizational level, capacity building for effective interventions to ensure sustainable development of biospheres will require a holistic approach, considering human needs. Such an approach will include strategic planning, human resource management, networking, professionalising and ensuring a conducive institutional environment for development of individuals, environmental groups and other stakeholder groups so that all interact with a shared vision for sustainable global development.

\section{Introduction}

Those gathered at the conference today will agree on the need to create more biosphere reserves and to protect those that we already have. The justifications for the Man and the Biosphere Reserves (MAB) established in several ecological settings are strong and accepted by this "community"*.. Any ecosystem has a relationship with a wide set of stakeholder groups whose needs and aspirations have a bearing on the creation, management and continuity of protected areas, such as, biospheres. The continuity of all species on planet earth and their associations with the different ecosystems depend on the latter's carrying capacity. The carrying capacity of the ecosystem is constantly being redefined by environment factors as well as by the influence of human interventions. Thus, in order to ensure that biospheres and other human endeavours are successful in achieving the long term goals, we need to examine the sustainability of such efforts.

The stakeholders associated with an ecosystem are a dynamic group. The direct primary users in the vicinity may be traditional users. Further, there may be a range of secondary users who are linked to these primary users. The development interventions and changing demography of an area, may with time, change the nature of the stakeholder groups. The interest groups are becoming more global in nature and the local population invariably loses power over the control of local resources. These dynamics need to be considered when deciding on strategies for capacity building for sustainable development.

The challenge for management is to balance the different needs of the stakeholders in a manner that their aspirations are met, so that the capacity of the entire ecosystem is not compromised. The range of stakeholders, both local and global, adds to the complexity of the factors, when one considers that the globe is a fragile system of interrelated ecosystems with even the micro-systems having an

"Those engaged directly to establish and develop biospheres. These persons will include the professionals, scientists, and policy makers, both local and international. They demarcate the territories for "protection". The people who may have traditionally used the area may or may not participate in the endeavours of the professionals. 
influence on the whole. Thus, deforestation in a certain location affects the weather patterns of other areas, the pollutants of one location have far reaching effects on distant areas. Thus, the local effects and their control, are influenced by stakeholders elsewhere. Agenda 21, adopted at the Earth Summit held in Rio, enshrines the primacy of people's needs, particularly focussing on poverty and deprivation that is the lot of the majority. The declaration also recognizes the intricate connection amongst several entities for sustained development that will ensure intergenerational equity while ensuring the entitlement of present generations to a healthy, productive, and peaceful life. Ensuring the effectiveness of the commitments made is a challenge in decision making, policy formulation and management of institutions responsible at the global, national and community levels.

The paper examines the issues of development and its sustainability, stakeholders in biosphere reserves, strategies for sustained development and the capacity of institutions to address development in a sustained and meaningful manner.

\section{What is Development?}

What is meant by development at a societal level has been debated considerably and the definitions have evolved and improved over time. The Human Development Report of the UNDP which presents the status of all countries in the world, has been consistently refined to make the concept encompass universally emerging consensus on what we mean by development. The understanding of development and the acceptable criteria has improved greatly and changed from the initial economic reductionist measures based on incomes. Development is a generic term that can be applied to technology, institutions, and organisations, however, at a societal level, the reference is made to human development, an inclusive concept, against which all state and non-state interventions are assessed when finding out the effects that such endeavours have had on society.

The core areas of focus of human development are poverty, inequality, human survival and environmental degradation. However, it is well recognized that no extent of development measured by these indicators is of relevance in the absence of freedom - thus, the inclusion of the human freedom index as a measure of human development in recent times. ${ }^{8}$

Some aspects of human well-being considered when measuring human development are as follows:

$\begin{array}{lll}\text { Education } & \text { Health } & \text { Population } \\ \text { Employment } & \text { Human freedom } & \text { Rural and urban disparities } \\ \text { Environment } & \text { Income and its distribution } & \text { Social security } \\ \text { Food and nutrition } & \text { Life expectancy } & \text { Status of children /women }\end{array}$


The above indicates the complexity of the concept of human development. These aspects have bearings and implications for different social institutions. Thus, in order to ensure that these aspects are equally well developed, the effectiveness of those institutions responsible for the levels of development of each of these components, needs to be dealt with. Thus, the need for multi-sectoral integrated approaches to development intervention. The limitations or lack of emphasis of one can pull down the development levels of a society in that aspect and have a negative effect on the overall development status of a community.

\section{Issues of Concern}

When examining sustainable development and capacity building, focussing on a few major issues that provide a frame of reference for the longterm vision for a desired state is helpful. The following five issues, presented in summary below, could provide the frame of reference.

\section{Environment degradation and externalities}

Concerns about the rapid depletion of natural resources, such as soil and water as well as arable land, have been expressed over the past few decades, both at the global as well as the regional and national levels. The depletion of resources has also been related to pollution of the air, waterways, the sea as well as the stratosphere. Indiscriminate dumping of waste from urban areas, industrial establishments, as well as toxic emissions from automobiles, industrial establishments, agricultural enterprises, etc., has been highlighted. Further, the toxic emissions or pollution that may happen in one location has far reaching implications and effects on locations far away. The continuity of these practices is not acceptable and needs to be managed in order to ensure a better quality of life for the people of today as well as for those of the future.

Significant environment degradation is caused by poverty in the developing countries and by affluence in the developed countries. ${ }^{7}$ Poverty and increasing population in developing countries force people to cultivate ever more marginalised lands. This erodes the soil further, making it less productive and making the people poorer. Women spend more time collecting firewood and water due to the destruction of the watersheds. Three quarters of people in developing countries live in ecologically fragile zones and around 1.4 million have become ecological refugees driven from their homes by ecological degradation. The average person in a developing country consumes only one third of the energy resources consumed by a person in a developed country. Energy consumption in gigajules is 30 in Latin America, 21 in Asia and 12 in Africa, while in some African countries it is less than one. On the other hand, Japan consumes 110, the Federal Republic of Germany 165 and USA 280. Further, the developed countries also produce six billion tonnes of greenhouse gases emitted each year, even though they hold only one fifth of the world's population? 


\section{Poverty and deprivation}

Global figures of poverty are alarming. The majority of people in the globe today continue to live below internationally accepted standards of poverty. Global disparities and intra-country disparities of income distribution and levels of poverty continue to alarm development economists. In many of the major regions known to have high levels of poverty, such as in South Asia and Africa, there have been improvements made in income distribution. Yet, widespread famine, conflicts and war, have brought misery to millions of people in these areas.

Poverty is more than a matter of low incomes. It is also capacity deprivation, where communities are made ineffective and excluded from the decision-making processes that affect their lives. ${ }^{6}$ Thus, the socio-political decision-making processes are determined for them by external agencies, resulting in effective exclusion. This situation results in decisions being taken that may be at the expense of their interests. The rural communities may find themselves in situations where local resources are demarcated as inaccessible to them. Such decisions may be detrimental to their well-being, although rationalised on the basis of wider and greater good of all people. More equitable and just arrangements for resource use may, thus, be possible if people were in a position to articulate their needs and concerns more effectively. This does not simply require their presence at the decision-making processes but requires the capacity for meaningful dialogue, bargaining, and total engagement.

\section{Population and consumption patterns}

The trends in population increases have been brought under control in most countries. The increase in populations, particularly among the poor nations as well as in regions that are already suffering due to resource limitations, continues to be a concern for demographers and development agencies.

The major cause of continued deterioration of the global environment is the unsustainable patterns of consumption and production systems. The industrialised countries have the highest consumption rates of energy and other resources and contribute more to the pollution of planet earth..$^{8,4}$ Yet, in the developing countries, expanding populations put more pressure on fragile ecosystems. More land is brought under cultivation, resulting in soil erosion and low productivity; triggering off a vicious cycle of need, land clearing, low productivity, more clearing of new land, and further soil erosion. Increasing populations and unabated consumption patterns in affluent societies are putting tremendous pressure on planet earth's fragile ecosystem.

Poverty reduces the people's ability to use resources in a sustainable manner, intensifying pressures on the ecosystem. ${ }^{8}$ Thus, the call for development to move 
from being material and energy intensive to a more equitable distribution of the benefits of development and resource use.

\section{Economic inequalities}

Economic inequality in all countries, both developed and developing, is of major concern partly due to the social tensions they create as well as to the ethical dilemmas faced. There are increasing disparities in affluence and deprivation due to unabated consumption patterns the world over. Changing weather patterns, poor productivity of soil, lack of arable land and depletion of available water, contribute to deteriorating living conditions for the poor in developing countries. War and strife as well as poor management of economies put further strain on ecosystems, resulting in people migrating as refugees and attempting to make a subsistence living on the land. Despite technological advancements, a fifth of the population in developing countries is left hungry, a quarter does not have safe drinking water, and a third live in abject poverty. ${ }^{8}$

\section{Good governance}

The ability of a nation to address the needs of its people in an acceptable manner is related to good governance. The concerns for good governance have in recent years taken centre stage in development discussions. This is due to the pivotal role that the state plays in the development efforts of a country. The government has an allpervasive influence on all facets of life, the absorption of social functions from traditional institutions, such as the family, community and religion. Being transparent, accountable and efficient in planning and implementing development interventions is required by those advocating good governance. It further incorporates respect for human rights and sustainability of development and equity of resource distribution and economic opportunities. Thus, the state is expected to play a more efficient role, free of corruption and inefficiencies. Such expectations of responsibility to the wider society are now being expected of the corporate sector too. Thus, the public and international discourse call for good governance, and they have a bearing on efforts towards sustainable development interventions.

\section{Sustainable development}

Sustainability is a concept about which social and natural scientists and philosophers have expressed their views. The views are diverse and varied, and continue to be debated upon. It has meant conservation of resources, population management, ensuring optimum carrying capacity in ecosystems, maintaining biodiversity, maintaining cultural diversity and social harmony, the inevitable dominant role of the state, and intergeneration equity. Economists, ecologists, sociologists, environmentalists, political scientists, management experts have all contributed to the debate and understanding of the complexities associated with sustainability 
and development. The focus on intergenerational equity advocated by economists has been accepted universally by intellectuals and policy makers. ${ }^{3}$

The diversity of views raises a major question. What is to be sustained? Sustainability of consumption or income levels or else the sustainability of the ecosystem? As stated by Chopra and Kadekodi, eco-fundamentalists will plead for resource sustainability, which in turn force the acceptance of a level of well-being that will ensure sustainability. The sustainability of ecosystems does not necessarily generate the highest levels of living. Accepting levels of living that may not be high, for the sake of future generations, depends on the moral restraints and value judgements of the present generations. This also depends on the institution's ability to deliver the benefits on the basis of equity and justice. Sustainable incomes which may also be defined as sustainable levels of living is thus an alternative paradigm. This will be the highest level of consumption that a present generation may choose to allow future generations to enjoy at least at present rates, if not higher.

A reconciliation of the diverse points of views is suggested by the World Commission on Environment and Development. "In essence, sustainable development is a process of change in which the exploitation of resources, the direction of investments, the orientation of technological development, and institutional change are all in harmony and enhances both current and future potential to meet human needs and aspirations". Such an opinion of sustainable development leaves room for adjustment of the following;

(i) institutional factors which will include markets, community and the state

(ii) economic factors such as investments

(iii) scientific and technological factors

(iv) people's participation in articulating that their needs and aspirations be met

The Rio Declaration of the Earth Summit recognised the rights of future generations, the entitlements of the present generations for a quality life as well as the inseparable connection between environment protection and economic progress. Eradicating poverty and reducing disparities in living standards in different parts of the world are also recognised as being essential for sustainable development and for meeting the needs of the majority of people. ${ }^{4}$

Sustainable development can be seen from a different perspective too. The concept of debt is used by the UNDP to assess sustainability and the responsibilities of the state. Thus, the following types of debt are incurred by governments that have a negative bearing on sustainable development: 
(i) Financial _ - borrowing heavily both externally and domestically

(ii) Social - by neglecting to invest in human development

(iii) Demographic - by permitting unchecked population growth

(iv) Environmental - by exhausting natural resources or polluting the land, water and air.

An overarching sector will be the institutions that will ensure the meeting of the requirements of all of the four sectors mentioned above.

\section{Institutional Frame for Sustainable Development}

Meaningful and sustained development requires the concerted efforts of a multitude of institutions and organisations, managed effectively by skilled persons. Development planning and interventions are no longer the exclusive concern of the state. Many non-state actors are recognised for their roles and are on board the efforts where the state still continues to play an important role. The key stakeholders can be listed as:

- Public sector - the policy makers, bureaucracy, judiciary and law enforcement agencies.

- Business and industries

- Women

- Children and young people

- Indigenous people

- Nongovernment organisations

- Local authorities

- Workers and trade unions

- Scientists and technologists

- Farmers

- Financial institutions

- Media

- Educational and training institutions

- Religious organisations

For the implementation of Agenda 21, several of these key stakeholder groups were identified. Yet, in the effective planning and implementation of strategies, a wider cross section of agencies are required to ensure success.

The stakeholders represent different interest groups or sectors. These agencies and sectors are interrelated in many different ways and form networks of institutions that have a bearing on the well-being of people and development efforts. Each entity in the network has a bearing on the other and the success of each depends on the effectiveness of the other and the collective drive towards a common goal. They need to work in unison and for the common goal of sustainable development. Such a definition of future goals must be arrived at through a process of consensus building.

In a network of organisations or institutions, each of the entities or partners will operate or function at different levels - local, regional, national or international. Those at each of the different levels may form horizontal networks, while those that may link across strata may form vertically linked networks. For example, at the 
local level in a catchment area, the community based organisations, such as the village development society may link with nongovernmental organisations (NGOs) as well as social organisations, such as the temple society and local-government officials to implement a catchment area protection plan. Such local level initiatives and actions require the collaboration of all related locally operating organisations and institutions. The same is true at district, provincial or state level organisations. In certain instances, it is necessary for the local organisation(s) to link up with the regional and national organisations for effective interventions. ${ }^{10}$

The key stakeholders listed above may belong to several different types of organisations and institutions. They will also be operating at different levels of development. The level of development of an organisation will depend on its ability to achieve its goals effectively and efficiently. As organisations develop, they show increasing differentiation and adaptation to the external environment and also specialisation in functions. ${ }^{5}$ Thus, organisational development will be characterised, among others, by the following:

(i) Clearly defined goals and the means to achieve them.

(ii) Clearly defined roles and expectations of the persons performing such roles.

(iii) Human resources in adequate numbers who are skilled and perform the assigned tasks at expected standards.

(iv) Effective recruitment, retention and retirement policy and strategies.

(v) Well defined standards of performance.

(vi) Human capital development strategy for longterm success in achieving goals, i.e., training.

(vii) Adequate resource dispersion and longterm investment plans.

(viii) Monitoring and evaluation procedure and mechanisms.

(ix) A functionally effective hierarchy and lines of command that are conducive for efficient and quick decision-making.

(x) Learning culture that enables both the organisation and the staff to constantly update their knowledge and skills to enable them to adapt effectively to a rapidly changing external environment. Thus, a strategy for the management of ideas that may come from any level of the organisation. 
(xi) Use of appropriate technology to ensure efficiency, effectiveness and quality of performance and output.

(xii) Adherence to high professional standards and constantly ensuring that a competitive edge over similar organisation is achieved.

(xiii) Ensuring that the organisation and its employees uphold high ethical standards in their conduct.

(xiv) Forming strategic alliances with other organisations for longterm sustainable development.

A complex interconnected network of organisations and institutions, as well as collectives of people make up society. The structure of society is complex due to the multiple roles all people play and the membership they have in formal and informal groups. The effectiveness with which a society is able to address its needs depends on the manner in which these organisations and institutions function and relate to each other. Sustainable development that is considerate of the environmental effects also calls for these organisations to be able to learn and be sensitive to the wider concerns of society that goes beyond the boundaries of their own goals and targets. Thus, the ability to recognise the needs of other entities is essential. Such an openness and efficacy calls for sustainable democracy within the network of organisations or society in general. ${ }^{2}$ Effective management of ecological and environmental concerns can be successful in the longterm, if organisations and society incorporate democratic principles of management in a sustained manner. This calls for a change in values and adoption of behavioural norms that will ensure incorporation of democratic principles at all levels of decision-making. Such efforts are wider than the capacity building interventions identified above or the simple exercise of franchise or participation in decision-making.

\section{Managing Organisations Effectively}

Strategic management is a widely introduced tool in the corporate sector as a means of adapting to a rapidly changing world with competing interests. The future of organisation management has to take into cognisance many trends and real changes $^{* *}$. Those that adapt and adjust to these trends will be more successful in a

\footnotetext{
"* The shift in strategic resources from an individual to an information society, where the strategic resource is its employees who possess information, knowledge and creativity. The emerging sellers market and the new competition is for the best employees. The whittling away of middle management with the computer making fewer people do many tasks. The continuing entrepreneurial revolution, the workforce becoming younger, better educated and increasingly female. Women reinventing career patterns and motherhood and lifestyles. The growing use of intuition and vision. The mismatch between the educational system and the needs of the new information society. The rising importance of corporate health issues. The values of a generation of managers that are educated, socially liberal, and health conscious.
} 
competitive economic environment. Further, a shift in philosophy is widely adopted as a means of survival and for maintaining a competitive edge. Strategic management includes three major dimensions.

Strategic analysis is considered the foundation of strategic management. The first dimension is the examination of the organisation's strategic goals in the light of external opportunities and threats. Being aware of the external environment is extremely crucial. If required, the goals are revised accordingly. The strategic goals are defined in congruence with the vision and mission of the organisation. Thus, they are ensured as being rational and also externally valid. The goals are also examined in the light of internal strengths and weaknesses. This ensures that the organisation has the potential capability of achieving its goals. The process ensures that the organisation does not set itself unrealistic goals.

The second dimension - strategy formulation - requires working at four levels - functional, business, corporate, and international. Thus, the basis of working and planning in an organisation becomes broader requiring the skills and abilities to address these issues effectively.

The third dimension of strategy implementation is the process of transformation of intentions into realities. This process consists of four components:

(i) Integration - The various sections of an organisation must work in sync with each other. The workers of all divisions interact and work towards a common goal.

(ii) Organisational structures - Strategy implementation requires effective and smooth communication among all personnel. The structure of the organisation should be conducive to free communication, exchange of information and informed decision making.

(iii) Strategic controls - Controls ensure that the efforts are "on track" and the necessary feedback is obtained to ensure that all activities and functions are focussed on achieving organisational goals.

(iv) Strategic leadership - Leadership influences all other aspects of strategy implementation. Leaders design structures and the required controls for organisations and are the driving force behind organisations.

\section{Organisational and Individual Capacity}

Organisations and individuals working therein are required to develop their capacities in order to be able to function effectively for sustained development. These efforts may be at a macro societal level, and may require the interventions of 
governments as well as international organizations and initiatives. Policy formulation and their effective implementation and collaboration among countries provide the broad macro situation within which the organisations and individuals will function. The macro issues have a bearing on the effectiveness of the efforts of sub-national groups or individuals. However, sub-national interest groups can have a bearing and influence on the manner in which national or international decisionmaking takes place or on how structures are developed.

At an organisational level, several aspects need strengthening in order to develop capacities for the sustainable development of organisations which in turn will ensure sustained development of their communities, countries, and the eco-system

i. The first of these is the stock of skills \& knowledge in the organisation, held by each of the persons working for them. The people who work make the organisation effective. The effectiveness and efficiency with which tasks are carried out depend on the skill levels of the individuals as well as on the work environment and culture of the organisation. The leadership structure and quality within the organisation also determine, to a great extent, the effectiveness of the employees. It is the totality of the employees, the quality of interpersonal relationships, as well as the leadership, that determines the effectiveness of the organisation and its ability to set and achieve goals and targets. Individuals and organisations need to develop the ability to be introspective and ask the right questions from themselves in order to develop better standards of performance to ensure longterm success. Such a capability needs to be developed through training for skills development and mainstreamed into the organisation's functioning. Introspection will motivate persons to develop their knowledge in a particular area of work or specialisation in order to keep abreast of matters. The organisation itself will have to provide the environment and resources to make this process effective.

ii. Sustained effectiveness of organisations or groups depends on the skills they possess. With changes in the external environment as well as the challenges from within, new skills and know-how are required. Training of varied kinds will be required to keep abreast of the challenges. Training can be self-learning, formal as well as informal, within or external to the organisations. The proper mix of intrinsic motivations and external stimuli can make individuals drive towards perfection through self-learning. Yet, formal, designed training programmes are required for specific capacity building needs of organisations.

iii. Most efforts at achieving goals of individuals or organisations cannot be met effectively by working in isolation. Networking with others who are similar, as well as with other stakeholders can strengthen the efforts and be more effective. This synergetic effect is recognised and used effectively today. 
Forming networks with appropriate entities is a strategic requirement. Such networks can be made formal and alliances developed for longterm sustained joint efforts. Such alliances may also be where there is an agreed division of labour recognising the strengths of each party and using such capabilities without replicating and increasing costs and running the risk of inefficiencies. Such collaborations where the services of one organisation are used by another, are differentiation, specialisation and functional re-integration depicting a high level of organisational development. Individuals should be able to recognise such opportunities and take action to make effect such ideas.

Any organisation that strives for perfection needs to take stock of what it is supposed to do and how it is to set about accomplishing its goals and targets. Such endeavours are routinely done. However, the rigour and degree of introspection and the wise use of information generated, may not be equally well-developed in all agencies and organisations. Close monitoring and evaluations of organisations are done routinely in the private sector, but not so rigorously in the government or NGO sector. For longterm success of organisations there is the need for striving for the best standards of professionalism. Such standards, while being unique to an organisation, will also have certain universal aspects. When one considers the modern world and the need for networking and developing strategic alliances; those that work for the networks would find it easy to function smoothly if they adhere to uniform standards of procedure and performance. Such standardisation efforts are inevitable for longterm sustained collaborations. Standards once set cannot be static, they need to be modified and improved depending on the responsiveness of the organisations to changes within as well as outside.

Exchange of ideas and experiences among persons enrich them further and create the space and opportunity for intellectual growth. The opportunities provided for persons of similar professional interests to meet or spend a required amount of time in a location different to her/his work place help develop a greater sensitivity to different work environments. Further, it provides opportunities for enhancing knowledge and provides other support systems for personal and professional growth. Such efforts will require the organisations to have effective communication policies and facilities as well as meetings and placements. Participation in professional seminars and annual sessions which are platforms for sharing experiences and knowledge are opportunities for learning as well as for professional networking and have the potential for the advancement of all concerned. Such efforts need to be designed with high standards in mind and with desired outcomes planned for.

All human endeavours require some form of resource outlay. Often these resources are obtained from different entities. Thus, there is a need to be accountable. Accountability also arises from being members of professional 
associations and by being public officials. In order to be accountable, persons must be aware of their obligations and use the methods available to ensure accountability. There is a responsibility on the part of the organisation as well as the wider society to ensure accountability of persons who work for them. Being accountable goes hand in hand with being ethical in one's conduct. Such codes of conduct for ethical well-being of persons and organisations need to be developed and adhered to. Participatory approaches to ensure that all members are involved in a consensus building process are important. Providing the appropriate means and instituting mechanisms to ensure transparency and accountability are requirements of organisations. Such improvements should be instituted to ensure sustainable development. The success of these efforts will depend on the skill and knowledge of the persons involved, as well as the quality of leadership provided to ensure success.

\section{Social Capacity}

Sustainable development where the dual concerns of ensuring sustainability of the environment as well as inter-generational equity are ensured, will require the development of social capacity of the entire society. Such social capacity can be built through several interrelated means.

There is a need for public engagement in the discourse and debate on management of natural resources. Such public engagement is not merely to keep them informed but also to obtain their active participation as equal members in discussions. This will require that the public is well informed, knowledgeable and articulate. Such capacity building has to be ensured if they are to be meaningfully engaged. Poor people who are disempowered and unable to assert their will are by nature unable to make any meaningful contribution to public discussions and debate. Thus, their exclusion is the result. Therefore, poverty alleviation which is not achievable in the short term, has to be a longterm goal. Therefore, a meaningful, inclusive situation of public engagement may remain an ideal to be achieved in the future. However, in the short term, interest groups and support systems can be a means of facilitating their participation and input into the discourse.

Social capacity for sustainable development requires that the processes of strengthening social institutions, groups and organisations are supported effectively. This support can come from the government, nongovernmental organisations as well as from the international community. The nature of the support can vary. This could be in terms of providing the policy and legal framework. Human resource development strategies that will ensure an adequate supply of skilled personnel to draw from, continuous training and skills development, ensuring that communication and other infrastructure facilities are developed, and a peaceful environment in which to function and grow are the other requirements. Monitoring and providing constructive feedback for improvements in the capabilities and programme 
intervention is also a critical need. The ability to manage the finances to ensure that sufficient resources are available for programmes for longterm sustainability requires organisational capacity and skills.

Public awareness and knowledge of issues related to sustainable development is required if public support is to be obtained for such efforts. When public funds are used and when the legislature is involved in legislating certain requirements, then the participation of the public is necessary. This requires public education and awareness programmes that are consistent. Public education programmes are usually not sustained and monitored for efficacy. Assessing the effectiveness of public education programmes needs to be undertaken periodically in order to make appropriate improvements.

Sustainable development as a social goal is achievable if there is widespread public acceptability of the notion. The sacrifice people will want to make in present consumption levels, in order to ensure that people elsewhere who live under terribly deprivable conditions are able to make a decent level of living or that future generations are able to consume at least the present levels of goods and services, and that the ecosystem is preserved at some acceptable level of "goodness", are all desirable states people need to accept. This acceptance will require a change in social values and norms of behaviour.

\section{Conclusions}

Sustainable development has different meanings. However, inter and intragenerational equity as well as ensuring a well managed ecosystem that will be able to sustain the needs of the people, are universally accepted as major dimensions of sustainable development.

The goals of sustainable development need to be articulated by society at large, so as to reflect the needs of all people. Once articulated, then the interventions at macro and micro levels must also be driven by the interests of the stakeholders and not limited to the interests of a few. Such endeavours will require the effective functioning of the various social institutions that work towards fulfilling various interests and needs of the people. The effective participation of the wider cross section of people will require that their capacity be developed. However, the large numbers of people who are in poverty are not capable of participating in this discourse in a meaningful manner. Thus, a prerequisite to societal engagement of these groups of people is eliminating of poverty and ensuring that they have a decent standard of living and are thus able to participate in the sustainable development discourse as equal partners and contribute positively to the deliberations of ensuring a better world order for all concerned. As a short term measure it is still necessary to get their needs factored into the debate on sustainable development. Thus, at a macro level there are certain requirements that need to be assured for sustainable 
development to be realised and related institutions to act effectively. Adopting appropriate policy measures and laws, and effectively implementing them, will provide the required macro framework for institutions to develop and implement strategies for sustainable development.

Building capacity needs to be done at different levels. At the societal level, it needs to be done through public awareness and education interventions. Such endeavours should be directed at educating the public as well as changing the values and norms of behaviour in terms of life goals and expectations as well as of consumption patterns and beliefs for the future.

Goals of organisations as well as of society should be redefined, incorporating emerging global consensus of equity, intergenerational justice, conservation and judicious use of resources, and limiting consumption. Good governance and the adoption of democratic principles of management at organisational and governmental levels, all require major shifts in values and norms. These will require tremendous investments in social infrastructure development to ensure sustainable development. Such sustainable development of social institutions and organisations, reinforce sustainable development efforts of other sectors. Longterm change in values and norms of behaviour will require programme interventions of a strategic nature targeted by specific systems, such as education and training as well as the media. Building the capacity of society is required to get their needs and aspirations into the efforts put to develop policies and programmes to ensure sustainable development.

At an organisational level too, the work culture needs to be changed to incorporate and mainstream efforts to ensure an institutionalised approach to achieve sustainable development. Such efforts at capacity building will include adopting appropriate management techniques and strategies to ensure good governance and longterm effectiveness. Managing people and ideas and ensuring their fruition in order to achieve organisational goals, is a challenge faced by those in senior management positions and policy levels. Developing a learning culture within which an organisation creates the environment to draw the best of the personnel is essential. When each organisation, or group, attempts to meet the challenges and adapt to be more effective, appropriate leadership becomes an essential factor.

Having appropriate human resource management plans, networking and forming strategic alliances with other stakeholders interested in sustainable development, adopting good work practices for monitoring and building the capabilities of people and providing the space and opportunity for people with the ability to perform in a dynamic world, are challenges faced by organisations and management. Dealing effectively with these challenges will require more than a change in mindset. Such changes will require management to have a reorientation of working principles as well as develop new skills to deal with the emerging 
challenges. The support provided by international, national and partner organisations would enhance the ability to change and be more effectively responsive to the challenges of sustainable development.

\section{References}

1 Aburdene P. and Naisbitt J. (1994). Megatrends for Women: Women are Changing the World. Arrow Books Ltd., London.

2 Buell J. and DeLuca 'T. (1996). Sustainable Democracy: Individuality and the Politics of the Environment. SAGE Publications, New Delhi.

3 Chopra K. and Gopal K. K. (1999). Operationalising Sustainable development. Indo-Dutch Studies on Development Alternatives, Sage Publications, 22. New Delhi.

4 Keating M. (1993). The Earth Summit's Agenda for Change. Centre for Our Common Future, Geneva.

$5 \quad$ Plau P. M. (1977). Inequality and Heterogeneity: A Primary Theory of Social Structure. The Free Press, New York.

6 Sen A. (1999). Development as Freedom. : Oxford University Press, New Delhi.

7 UNDP (1991). Human Development Report. Oxford University Press, New York.

8 UNDP (1994). Human Development Report. Oxford University Press, New York.

9 UNDP (1997). Human Development Report. Oxford University Press, New York.

10 Warren R. L. (1978). The Community in America, Rand McNally College Publishing Company, Chicago. 